\title{
Hybrid Spline-Based Multimodal Registration Using Local Measures for Joint Entropy and Mutual Information
}

\author{
Andreas Biesdorf ${ }^{1}$, Stefan Wörz ${ }^{1}$, Hans-Jürgen Kaiser ${ }^{2}$, Christoph Stippich ${ }^{3}$, \\ and Karl Rohr ${ }^{1}$ \\ ${ }^{1}$ University of Heidelberg, BIOQUANT, IPMB, and DKFZ Heidelberg \\ Dept. Bioinformatics and Functional Genomics, Biomedical Computer Vision Group \\ ${ }^{2}$ University Hospital, RWTH Aachen University, Dept. of Nuclear Medicine \\ ${ }^{3}$ University Hospital Heidelberg, Dept. of Neuroradiology
}

\begin{abstract}
We introduce a new hybrid approach for spline-based elastic registration of multimodal medical images. The approach uses point landmarks as well as intensity information based on local analytic measures for joint entropy and mutual information. The information-theoretic similarity measures are computationally efficient and can be optimized independently for each voxel. We have applied our approach to synthetic images, brain phantom images, as well as clinically relevant multimodal medical images. We also compared our measures with previous measures.
\end{abstract}

\section{Introduction}

Image registration is an important technique for medical diagnosis, surgical planning, and treatment control. A challenge is to cope with the broad range of applications as well as the large spectrum of imaging modalities. Previous approaches for image registration can be classified according to the transformation model (e.g., rigid or elastic) and the used image information (e.g., landmarks or intensities). Landmark-based approaches are computationally efficient and allow coping with large geometric differences, while intensity-based approaches use more image information and do not require segmentation. Concerning intensity-based approaches, it is important to distinguish between monomodal and multimodal registration problems, since different types of similarity metrics are required. Monomodal registration can be achieved by, e.g., using the sum of squared intensity differences (SSD). Registration of images of different modalities, however, requires multimodal similarity metrics such as joint entropy (JE) or mutual information (MI). In general, registration using such information-theoretic intensity similarity metrics is robust and accurate, however, these metrics are computationally expensive since they require the estimation of probability density functions based on joint histograms (e.g., [1/23]).

In recent years, increased attention has been paid to hybrid registration approaches that integrate both landmark and intensity information (e.g., 4 456767891011$]$ ). Hybrid approaches can be classified into two classes. The first 
class of approaches uses the two types of information subsequently. Typically, one type of information is used to perform an affine preregistration to decrease computation time in the following elastic registration step (e.g., 456678]). The second class of hybrid approaches couples both types of information directly (e.g., 91011]). However, only few hybrid approaches have been designed for multimodal images (e.g., 9577) and, even if information-theoretic similarity measures are employed, often only application to monomodal images is reported. In addition, previous spline-based hybrid approaches typically use coarse physical deformation models such as B-splines (e.g., 977]) or thin-plate splines (e.g., 58]), and incorporate intensity information using global, computationally expensive information-theoretic measures. Local information theoretic measures exists (e.g., [1213]), however, they have not been used in hybrid registration schemes.

In this contribution, we present a new hybrid approach for spline-based elastic registration of multimodal medical images. Our approach is formulated as an energy-minimizing functional that incorporates point landmarks and intensity information as well as a regularization using physically-based splines. The intensity information is evaluated locally based on analytic measures for joint entropy and mutual information. We suggest new information-theoretic measures that are modifications of the measures in [13], but which are more robust w.r.t. noise as well as more similar to the original information-theoretic formulations. In contrast to 13 , where the measures are evaluated globally, we here evaluate and optimize the measures locally, i.e., independently for each voxel, and integrate them within a hybrid spline-based registration scheme.

\section{Multimodal Hybrid Elastic Registration}

\subsection{Hybrid Energy Minimizing Functional}

Our hybrid approach for elastic registration of multimodal images is based on an energy minimizing functional $J_{\text {Hybrid }}$ that incorporates both landmark and intensity information and a regularization term:

$$
J_{\text {Hybrid }}(\mathbf{u})=J_{\text {Data }, I}\left(g_{1}, g_{2}, \mathbf{u}^{I}\right)+\lambda_{I} J_{I}\left(\mathbf{u}, \mathbf{u}^{I}\right)+\lambda_{L} J_{\text {Data }, L}(\mathbf{u})+\lambda_{E} J_{E l}(\mathbf{u})
$$

The first term $J_{\text {Data,I }}$ describes the intensity-based similarity measure between the source and target image, $g_{1}$ and $g_{2}$, respectively. With the second term $J_{I}$ the intensity-based deformation field $\mathbf{u}^{I}$ is coupled with the final deformation field $\mathbf{u}$ using a weighted Euclidean distance. The term $J_{\text {Data }, L}$ incorporates the landmark information based on approximating Gaussian Elastic Body Splines (GEBS). In contrast to other splines, an advantage of GEBS is that cross-effects can be handled [14, i.e., transversal contractions lead to longitudinal dilations according to properties of elastic tissue. The fourth term $J_{E l}$ represents the regularization of the deformation field according to the Navier equation of linear elasticity, which constrains the deformation field to physically realistic deformations. The overall functional $J_{\text {Hybrid }}$ is minimized alternatingly w.r.t. $\mathbf{u}^{I}$ and $\mathbf{u}$. 
In previous work [11], $J_{\text {Data,I }}$ was defined based on the sum-of-squared intensity differences, thus the approach was only applicable to monomodal registration. Here, we present two new formulations of $J_{\text {Data,I }}$ for multimodal registration as well as new schemes for minimizing $J_{\text {Hybrid }}$ w.r.t. $\mathbf{u}^{I}$.

\subsection{Multimodal Registration Using Analytic Measures for Joint Entropy and Mutual Information}

For the intensity-based similarity measure $J_{\text {Data,I }}$ in (1) we suggest to use local analytic measures for joint entropy and mutual information. Let $g$ be an image of dimension $d$ over the continuous domain $\Omega \subset \mathbb{R}^{d}$, and $N_{R}(\mathbf{x})$ be the neighborhood of radius $R$ around a point $\mathrm{x} \in \Omega$. For $R$ being sufficiently small, the first order Taylor approximation $T_{g}(\mathbf{x}) \simeq \nabla g(\mathbf{x})^{T} \cdot \mathbf{x}+g_{0}(\mathbf{x})$ can be used as an approximation of $g(\mathbf{x})$ in the neighborhood of $\mathbf{x}$, where $\nabla g$ denotes the image gradient. The intensities $g(\mathbf{x})$ in the neighborhood $N_{R}(\mathbf{x})$ can be characterized by a random variable $\mathbf{g}$, which can be described by a probability density function. In 13 it was shown that for two images $g_{1}$ and $g_{2}$ the joint entropy $J E(\mathbf{x})$ and the mutual information $M I(\mathbf{x})$ can then be approximated by

$$
\begin{aligned}
& J E_{\text {orig }}(\mathbf{x}):\left\{c_{d}+\log _{2}\left(\left\|\nabla g_{1}(\mathbf{x})\right\|\left\|\nabla g_{2}(\mathbf{x})\right\||\sin (\theta)|\right)\right\} \rightarrow \min , \\
& M I_{\text {orig }}(\mathbf{x}):\left\{c_{d}-\log _{2}(|\sin \theta|)\right\} \rightarrow \max ,
\end{aligned}
$$

where $\theta$ represents the angle between $\nabla g_{1}(\mathbf{x})$ and $\nabla g_{2}(\mathbf{x}), c_{d}$ is a constant that depends on the dimension $d$, and $\|\cdot\|$ denotes the Euclidean norm. However, due to properties of the logarithm, $J E_{\text {orig }}(\mathbf{x})$ and $M I_{\text {orig }}(\mathbf{x})$ yield very large absolute values if the argument $\left\|\nabla g_{1}(\mathbf{x})\right\|\left\|\nabla g_{2}(\mathbf{x})\right\||\sin (\theta)|$ or $|\sin \theta|$ is close to zero which leads to unstable results. In [13], $J E_{\text {orig }}(\mathbf{x})$ and $M I_{\text {orig }}(\mathbf{x})$ were evaluated globally, i.e., the similarity for each voxel is integrated over the whole image. If, however, at a single voxel the argument of the logarithm is zero or close to zero, the global measure yields a very large value, since adding logarithmic terms is equivalent to the logarithm of the product of the arguments $\left(\sum_{i} \log _{2} s_{i}=\log _{2} \prod_{i} s_{i}\right)$. Due to the singularity of the logarithm, the value at a single voxel can render the result of the metric useless. To circumvent this problem, in 13. simplified metrics have been used which omit the logarithm:

$$
\begin{aligned}
& J E_{\text {simp }}(\mathbf{x}):\left\{\left(\nabla g_{1}^{T}(\mathbf{x}) \nabla g_{2}(\mathbf{x})\right)^{2}\right\} \rightarrow \max \\
& M I_{\text {simp }}(\mathbf{x}):\left\{\cos ^{2} \theta\right\} \rightarrow \max
\end{aligned}
$$

A disadvantage of these measures is that they differ largely from the original measures in (2) and (3). Instead, we here propose alternative formulations for $J E$ and $M I$ that do not have a singularity and that are more similar to the original measures.

Joint Entropy Measure. For JE, we suggest an alternative measure to (2), where the logarithm is still included. To illustrate and motivate this modification, we have generated synthetic images simulating a multimodal registration 


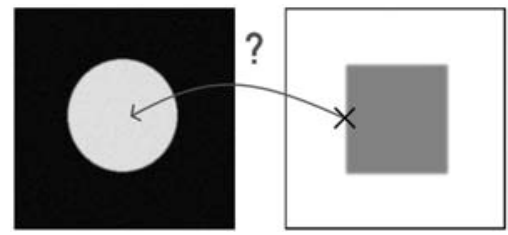

Fig. 1. Registration problem: Source and target images

problem (light circle on a dark background and dark square on a light background, Fig. 11). By registration, the circle has to be stretched to the size and shape of the square. For each point in the source image, we have computed the similarity values w.r.t. the marked reference point (black cross) in the target image and have visualized them as 3D plots in Fig. 2. In each of the plots, the intended optimum is marked with a black arrow and circle. In (a), the result for $J E_{\text {orig }}(\mathbf{x})$ is shown. Since $J E_{\text {orig }}(\mathbf{x})$ needs to be minimized, the optimum can be found at the saddle point between the two elevated semi-circles. Obviously, this minimum is difficult to identify since it has a similar value as the background, which is very noisy. To reduce the sensitivity to noise, we introduce a constant $\epsilon>0$, which is added to the argument. This leads to the result in (b). Now, the background noise is reduced significantly, but the optimum is still located at the saddle point between the two semi-circles, which is difficult to identify. To facilitate optimization, we replace $|\sin (\theta)|$ in (2) with $|\cos (\theta)|$, which leads to:

$$
J E_{\text {new }}(\mathbf{x}):\left\{\log _{2}\left(\epsilon+\left\|\nabla g_{1}(\mathbf{x})\right\|\left\|\nabla g_{2}(\mathbf{x})\right\||\cos (\theta)|\right)\right\} \rightarrow \max
$$

Now, the measure has to be maximized, which is much easier (see (c)) than finding the saddle point in (b).

Mutual Information Measure. For $M I$, we suggest a similar modification where the logarithm is still included and a constant $\epsilon>0$ is introduced. Here, the introduction of $\epsilon$ also improves the robustness of the metric:

$$
M I_{\text {new }}(\mathbf{x}):\left\{\log _{2}|\epsilon+\sin \theta|\right\} \rightarrow \min
$$

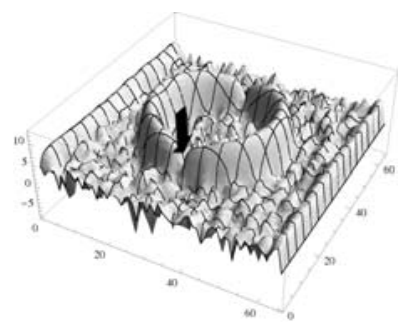

(a)

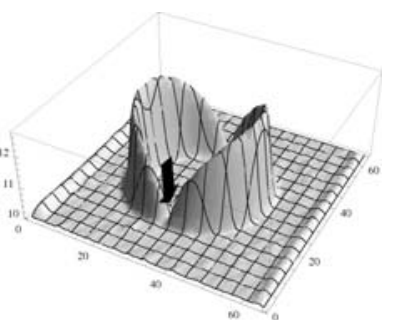

(b)

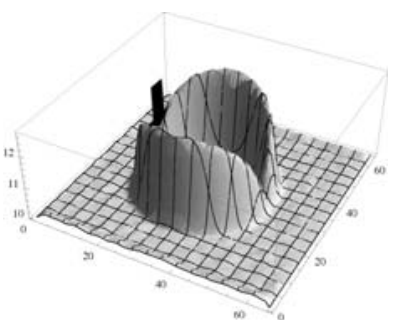

(c)

Fig. 2. Results for different metrics for $J E$ for registration of the images in Fig. 1 (a) $J E_{\text {orig }}(\mathbf{x})$, (b) $J E_{\text {orig }}(\mathbf{x})$ after introducing a constant $\epsilon$, (c) using $J E_{\text {new }}(\mathbf{x})$ 


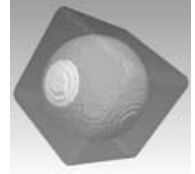

(a)

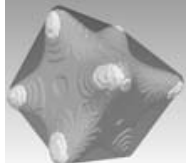

(b)

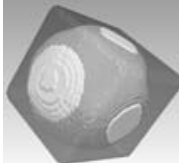

(c)

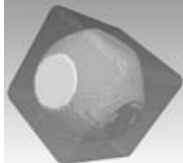

(d)

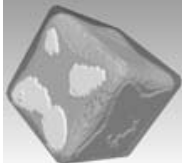

(e)

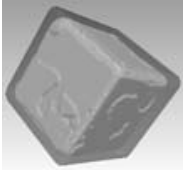

(f)

Fig. 3. Registration of a sphere with a cube. (a) Original source and target images, (b) after landmark-based registration, (c) after intensity-based registration using $J E_{n e w}$, (d) after intensity-based registration using $M I_{\text {new }}$, (e) after hybrid registration using $J E_{\text {new }}$, and (f) after hybrid registration using $M I_{\text {new }}$.

In our registration approach, the measures are integrated as $J_{\text {Data,I }}$ in (1). Optimization of $J_{\text {Hybrid }}$ is performed alternatingly w.r.t. $\mathbf{u}^{I}$ and $\mathbf{u}$. For minimization w.r.t. $\mathbf{u}^{I}, J_{\text {Data, } I}+\lambda_{I} J_{I}$ has to be minimized. To this end we have derived analytic expressions for the partial derivatives of the different measures. Note that in contrast to [13], in our approach $M I$ and $J E$ are evaluated locally, i.e., optimization of $\mathbf{u}^{I}$ is computed independently for each voxel, which improves the efficiency. In comparison to [11], we here use a minimization scheme based on steepest gradient since the analytic information-theoretic measures cannot be formulated by squared differences and thus the method of Levenberg-Marquardt cannot be used.

\section{Experimental Results}

We have applied our registration approach to 3D synthetic images, 3D brain phantom images, and clinically relevant 3D medical images. In a first experiment, we have applied our approach to the 3D synthetic sphere and cube shown in Fig. 3a. The images have a size of $128 \times 128 \times 128$ voxels and inverted contrast to simulate a multimodal registration problem. Thus, a monomodal similarity measure (e.g., SSD) would fail. Note that registration is difficult since the volume of the cube is nearly two times larger than the volume of the sphere. Eight landmarks were defined at the corners of the cube, and the registration result is computed based on the different metrics. Fig. 3 shows the results for landmarkbased registration (b), for intensity-based registration based on $J E_{\text {new }}$ (c) and $M I_{\text {new }}(\mathrm{d})$, as well as for hybrid registration based on $J E_{\text {new }}$ (e) and $M I_{\text {new }}$ (f). It can be seen that $J E_{\text {new }}$ and $M I_{\text {new }}$ yield similar results. For landmark-based registration, only the corners of the cube are aligned. Using intensity-based registration, the faces of the sphere are aligned, but not the corners. The best result is obtained using the new hybrid approach, since both the faces as well as the corners are aligned.

To compare the results using our new measures with those in $[13$, we evaluated the registration accuracy for intensity-based registration of multimodal phantom images (MRI-PD and MRI-T1) from the BrainWeb database [15] for different levels of Gaussian noise $\left(\sigma_{n}=0,5,9\right.$; see Fig. 4 a, b for the case of $\left.\sigma_{n}=5\right)$. Here, we present results for the comparison of $M I_{\text {new }}$ and $M I_{\text {simp }}$. We have generated 


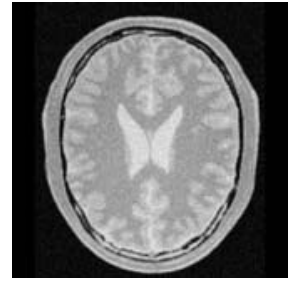

(a)

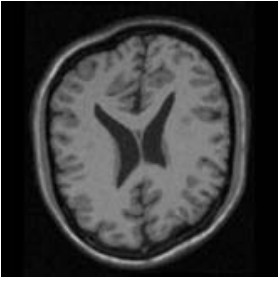

(b)

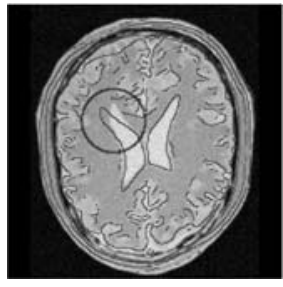

(c)

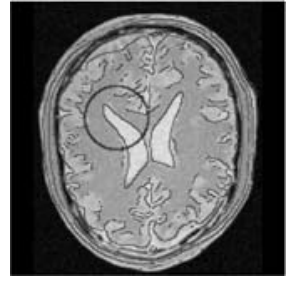

(d)

Fig. 4. Comparison of $M I_{\text {simp }}$ and $M I_{\text {new }}$ : (a) Source and (b) target image for $\sigma_{n}=5$. Registration result and edge overlay of the target image for (c) $M I_{\text {simp }}$ and (d) $M I_{\text {new }}$.

a deformation field $\mathbf{u}_{o}$ based on eight landmarks using GEBS, which is applied to the different MRI-T1 images to obtain three target images with known elastic deformations and different levels of noise. After registration, we compared the computed deformation $\mathbf{u}$ with the original deformation $\mathbf{u}_{o}$ and quantified the registration accuracy by the mean geometric error $\bar{e}_{g}=\overline{\left\|\mathbf{u}_{o}-\mathbf{u}\right\|}$. Note that in this experiment we did not use landmarks. Without registration, we have $\bar{e}_{g}=$ 3.40 pixels. For $\sigma_{n}=0$, registration using $M I_{\text {simp }}$ and $M I_{\text {new }}$ yields $\bar{e}_{g}=1.44$ and $\bar{e}_{g}=1.08$ pixels, respectively. With increasing level of noise, our new measure consistently yields more accurate results than the previous measure. Fig. 4 for example, shows the registration results for $\sigma_{n}=5$ for $M I_{\text {simp }}$ (c) and $M I_{n e w}$ (d). It can be seen that registration is more accurate in the neighborhood of the ventricles when using $M I_{n e w}$. The visual result agrees with the quantitative values: $\bar{e}_{g}=1.85$ for $M I_{\text {simp }}$, and $\bar{e}_{g}=1.55$ for $M I_{n e w}$. For $\sigma_{n}=9, M I_{\text {simp }}$ yields $\bar{e}_{g}=2.30$, while $M I_{\text {new }}$ yields $\bar{e}_{g}=1.78$. Thus, our new measure $M I_{\text {new }}$ is more robust w.r.t. noise than $M I_{\text {simp }}$. We also investigated the effect of $\epsilon$ on the registration result of $M I_{\text {new }}$. We found that the choice of $\epsilon$ is not critical, however, best results were obtained for $0.5 \leq \epsilon \leq 1.5$.

Furthermore, we quantified the registration accuracy of our approach using real, clinically relevant 3D multimodal medical images. We used five pairs of 3D PET transmission $(170 \times 128 \times 128$ voxels $)$ and $3 \mathrm{D} \mathrm{CT}(320 \times 400 \times 400$ voxels $)$ images of the human chest. Fig. 5, for example, shows two orthogonal slices of a 3D PET (a) and 3D CT (b) image before registration, as well as overlay images of the PET image with computed edges of the CT image before (c), after landmark-based (d), after intensity-based (e), and after hybrid registration (f) using $M I_{\text {new }}$. It can be seen that both the landmark-based and intensity-based registration schemes improve the alignment significantly, however, the best result

Table 1. Registration of 3D PET and CT images: Mean geometric error $\bar{e}_{g}$ (in voxel) for different metrics using different types of image information

\begin{tabular}{|c|c|c|c|c|c|c|c|c|c|c|}
\hline & & & \multicolumn{4}{|c|}{ Intensities } & \multicolumn{4}{|c|}{ Hybrid } \\
\hline & Unreg. & LM & $J E_{\text {simp }}$ & $J E_{n e u}$ & $M I_{\text {simp }}$ & $M I_{\text {new }}$ & $J E_{\operatorname{simp}}$ & $J E_{\text {new }}$ & $M I_{\text {simp }}$ & $M I_{\text {new }}$ \\
\hline$\overline{\bar{e}_{g}}$ & 7.36 & 5.32 & 5.60 & 5.76 & 6.70 & 5.60 & 5.17 & 5.05 & 5.28 & 4.83 \\
\hline
\end{tabular}




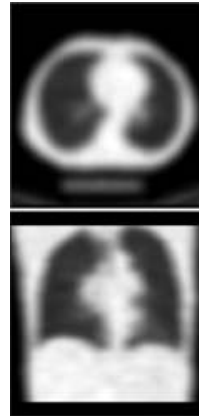

(a)

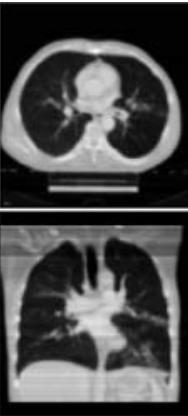

(b)

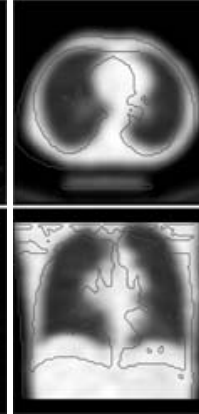

(c)

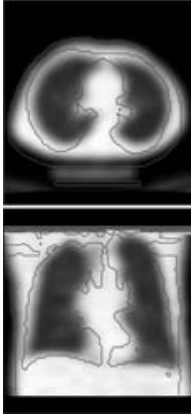

(d)

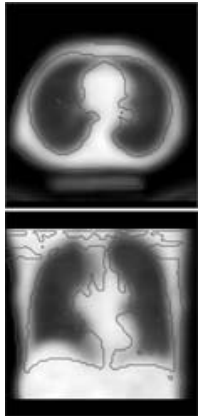

(e)

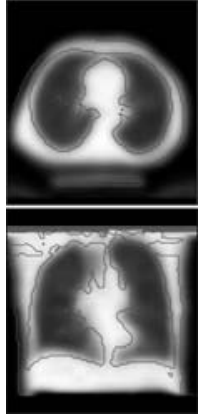

(f)

Fig. 5. Orthogonal slices of original (a) 3D PET transmission and (b) CT images. Source image with edge overlay of the target image (c) before, (d) after landmarkbased, (e) after intensity-based, and (f) after hybrid registration using $M I_{\text {new }}$

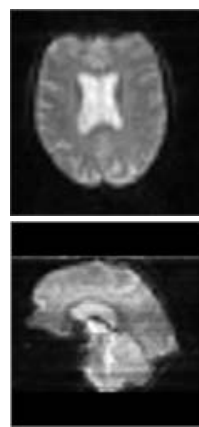

(a)

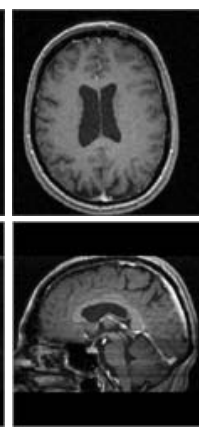

(b)

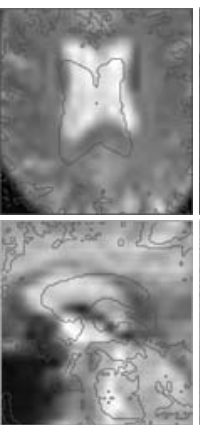

(c)

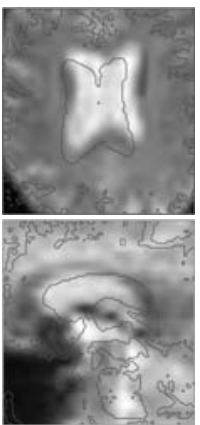

(d)

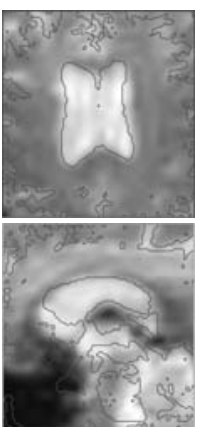

(e)

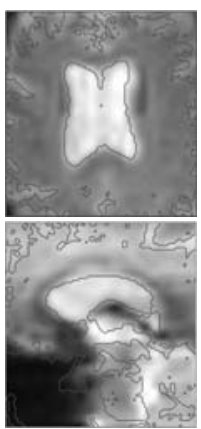

(f)

Fig. 6. Transversal and saggital slices of original (a) 3D fMRI and (b) MRI-T1 images. Section of the source image with edge overlay of the target image (c) before, (d) after landmark-based, (e) after intensity-based, and after (f) hybrid registration using $M I_{\text {new }}$.

is obtained when using the new hybrid scheme. This was also confirmed quantitatively. For this purpose, we identified ten pairs of corresponding anatomical point landmarks in each source and target image and computed the mean geometric registration error $\bar{e}_{g}$ (see Tab. 1). Before registration, we have $\bar{e}_{g}=7.36$ voxels. Using landmarks only, we have $\bar{e}_{g}=5.32$ voxels, which is a relative improvement of $27.7 \%$. Using intensity information only, we obtain $\bar{e}_{g}=5.60(23.8 \%)$ and $\bar{e}_{g}=5.76(21.7 \%)$ voxels for $J E_{\text {simp }}$ and $J E_{n e w}$, respectively. For $M I_{\text {simp }}$ and $M I_{\text {new }}$ we obtain $\bar{e}_{g}=6.70(9.0 \%)$ and $\bar{e}_{g}=5.60(24.0 \%)$ voxels. When using the hybrid approach, we obtain $\bar{e}_{g}=5.17(29.7 \%)$ for $J E_{\text {simp }}$ and $\bar{e}_{g}=$ $5.05(31.4 \%)$ for $J E_{n e w}$ as well as $\bar{e}_{g}=5.28(28.2 \%)$ for $M I_{\text {simp }}$ and $\bar{e}_{g}=$ $4.83(34.3 \%)$ for $M I_{n e w}$. Thus, in all cases, the hybrid approach is more accurate than the intensity-based and the landmark-based approaches. Furthermore, the 
new intensity similarity measures $J E_{\text {new }}$ and $M I_{\text {new }}$ yield more accurate results for the hybrid approach than the previous measures $J E_{\text {simp }}$ and $M I_{\text {simp }}$. Overall, $M I_{\text {new }}$ yields the best results.

We also applied our approach to register 3D MRI-T1 and functional MRI $(\mathrm{fMRI})$ images $(144 \times 256 \times 256$ voxels $)$. Fig. 6 shows a transversal and saggital view of 3D fMRI (a) and MRI-T1 (b) images. Before registration (c), a significant misalignment between source and target image is visible. After landmark-based registration using seven landmarks, a slight improvement is visible in the neighborhood of the ventricles (d). After intensity-based registration using $M I_{\text {new }}(\mathrm{e})$, the global alignment is improved significantly, although local misalignments still exist. The best result is obtained when using our new hybrid approach (f), since both the global as well as the local alignment is improved.

\section{Discussion}

We introduced a new hybrid spline-based approach for elastic registration of multimodal images. The approach incorporates point landmarks, intensity information, as well as a physically-based regularization. Since the approach uses new local analytic measures for mutual information and joint entropy, the required derivatives can be calculated analytically, and optimization can be performed independently for each voxel. We have demonstrated the applicability of our approach using 3D synthetic images, 3D phantom images, and different types of real 3D medical images. It turned out that our new similarity measures yield a more accurate registration result than previously proposed measures. We also found that the hybrid approach improves the results compared to a pure intensity-based and a pure landmark-based scheme.

Acknowledgments. Support of the Deutsche Forschungsgemeinschaft (DFG) within the project ELASTIR (RO 2471/2) is gratefully acknowledged.

\section{References}

1. Viola, P., Wells, W.M.: Alignment by maximization of mutual information. In: Proc. ICCV 1995, Boston, MA, USA, pp. 16-23. IEEE Computer Society Press, Los Alamitos (1995)

2. Maes, F., Collignon, A., Vandermeulen, D., Marchal, G., Suetens, P.: Multimodality image registration by maximization of mutual information. IEEE Trans. Med. Imaging 16(2), 187-198 (1997)

3. Rueckert, D., Sonoda, L., Hayes, C., Hill, D., Leach, M., Hawkes, D.: Nonrigid registration using free-form deformations: application to breast $\mathrm{mr}$ images. IEEE Trans. Med. Imaging 18(8), 712-721 (1999)

4. Johnson, H., Christensen, G.: Consistent landmark and intensity-based image registration. IEEE Trans. Med. Imag. 21(5), 450-461 (2002)

5. Wang, X., Feng, D.D.: Automatic Hybrid Registration for 2-D CT Abdominal Images. In: Proc. ICIG 2004, Hong Kong, China, pp. 208-211. IEEE Computer Society Press, Los Alamitos (2004) 
6. Rohr, K., Cathier, P., Wörz, S.: Elastic registration of electrophoresis images using intensity information and point landmarks. Pattern Recognition 37(5), 1035-1048 (2004)

7. Teng, C.-C., Shapiro, L.G., Kalet, I.: Head and neck lymph node region delineation using a hybrid image registration method. In: Proc. ISBI 2006, Arlington, VA, USA, pp. 462-465 (2006)

8. Azar, A., Xu, C., Pennec, X., Ayache, N.: An interactive hybrid non-rigid registration framework for $3 \mathrm{~d}$ medical images. In: Proc. ISBI 2006, Arlington, VA, USA, pp. 824-827 (2006)

9. Hartkens, T., Hill, D., Castellano-Smith, A., Hawkes, D., Maurer, C., Martin, A., Hall, W., Liu, H., Truwit, C.: Using points and surfaces to improve voxel-based nonrigid registration. In: Dohi, T., Kikinis, R. (eds.) MICCAI 2002. LNCS, vol. 2489, pp. 565-572. Springer, Heidelberg (2002)

10. Kybic, J., Unser, M.: Fast parametric elastic image registration. IEEE Trans Image Processing 12(11), 1427-1442 (2003)

11. Wörz, S., Rohr, K.: Hybrid Physics-Based Elastic Image Registration Using Approximating Splines. In: Proc. MI 2008: Image Processing, San Diego, CA/USA (2008)

12. Hermosillo, G., Chef d'Hotel, C., Faugeras, O.D.: Variational methods for multimodal image matching. Internat. J. of Computer Vision 50(3), 329-343 (2002)

13. Karaçali, B.: Information theoretic deformable registration using local image information. Internat. J. of Computer Vision 72, 219-237 (2007)

14. Wörz, S., Rohr, K.: Physics-based elastic registration using non-radial basis functions and including landmark localization uncertainties. Computer Vision and Image Understanding 111, 263-274 (2008)

15. Kwan, R.K.-S., Evans, A.C., Pike, G.B.: MRI simulation-based evaluation of imageprocessing and classification methods. IEEE Trans. Med. Imaging 18(11), 10851097 (1999) 